\title{
The Role of Glutamate Dehydrogenase Activity in Development of Neurodegenerative Disorders
}

\author{
Matej Kravos ${ }^{1 *}$, Ivan Malešič ${ }^{2}$ \\ ${ }^{1}$ University of Litoral, Koper, Slovenia \\ ${ }^{2}$ Faculty of Medicine, University of Maribor, Maribor, Slovenia \\ Email: ^mk4@siol.net
}

How to cite this paper: Kravos, M. and Malešič, I. (2017) The Role of Glutamate Dehydrogenase Activity in Development of Neurodegenerative Disorders. World Journal of Neuroscience, 7, 181-192. https://doi.org/10.4236/wjns.2017.71013

Received: October 19, 2016

Accepted: February 21, 2017

Published: February 24, 2017

Copyright $\odot 2017$ by authors and Scientific Research Publishing Inc. This work is licensed under the Creative Commons Attribution International License (CC BY 4.0).

http://creativecommons.org/licenses/by/4.0/

\begin{abstract}
The specific role of Glutamate dehydrogenase (GLDH) in the brain is not yet clear, but it is an important enzyme in protein degradation as well as a metabolism regulator of glutamate as a neurotransmitter. The enzyme probably provides crucial protection for postsynaptic membranes against the neurotoxic effects of glutamate neurotransmitters. In men, GLDH activity declines almost evenly through the ages; in women, it declines faster in the first five decades. In the years of menopause, GLDH activity declines slower. The diminished GLDH activities in leukocytes and in the brain vary considerably, but they are parallel with the progress of neurodegenerative diseases. The GLDH activity is partly deficient in the brain, particularly in the leukocytes of patients with heterogeneous neurological disorders and degeneration of multiple neuronal systems. We found a statistically significant difference of GLDH activity in the cerebrospinal fluid in patients with neurological diseases and unexpected in patients with degenerative and inflammatory disorders. The decrease in GLDH activity in the cerebrospinal fluid of patients with neurodegenerative disorders may be one of the reasons for the neuro-excitotoxic glutamate effect. Defining the GLDH activity in leukocytes is at the moment the sole experimental method. The second one could be the measurement in cerebrospinal fluid. The results suggest a possibility to regulate glutamate level in human brain through activation of GLDH.
\end{abstract}

\section{Keywords}

Glutamate Dehydrogenase, Neurodegenerative Disorders, Leukocyte GLDH, Cerebrospinal Fluid GLDH

\section{Introduction}

Glutamate dehydrogenase (GLDH) (EC. 1.4.1.3.) consists of only polypeptide 
chains. The active compound is a hexamer with molecular mass between 310,000 and 350,000 [1] [2]. GLDH has the key role of acting as an interface between carbohydrates and amino acids in the vicinity of the citric acid cycle and urea cycle in the metabolism of a cell. Its function is to catalyse reversible deamination of glutamate into $\alpha$-ketoglutarate and ammonium ion [2] [3] [4]. There are two forms: thermostable and thermolabile [1] [5] [6]. With thermal inactivation of serum, GLDH was found that almost one third of GLDH serum originated from the rough endoplasmic reticulum and the rest from mitochondria [4].

The specific role of GLDH in the brain is not yet clear, but it is an important enzyme in protein degradation [7]. It is linked with at least two sources of glutamate in the brain. In the synaptic part, glutamate acts as a neurotransmitter, while in the glial cells it acts as a metabolism regulator. Most of it is contained within astrocyte extensions [8]. The enzyme probably provides crucial protection for postsynaptic membranes against the neurotoxic effects of glutamate neurotransmitters [1] [9]. The glutamine metabolism in the brain is predominantly regulated by the control of GLDH rather than glutamine synthetase [10] [11]. However, glutamate has also been shown to have a role in the transduction of sensory input at the periphery, and in particular in nociceptive transmission [12] [13] [14].

The pathological glutamate excitotoxic mechanism in the brain is thought to be caused by processes, such as inadequate uptake, pathological metabolism in glial cells or release from terminals, and triggers excessive glutamate activity [2] [8]. Its excess is also implicated in peripheral neuropathies [15]. Studies of glutamate metabolism in astrocytes have shown that a considerable proportion of glutamate undergoes oxidative degradation [16]. Trauma in human beings, such as arthritis, myalgia, and tendonitis, elevates glutamate levels in affected tissues [17]. Excitatory amino-acid released from motoneuron terminals acts on metabotropic glutamate receptors on interneurons other than Renshaw cells [18].

\section{GLDH in Leukocytes}

Neutrophils contain all the enzymes including GLDH involved in the main metabolic pathways [19]. GLDH stimulates polyclonal proliferation and differentiation of naïve B-cells and, consecutively, immunoglobulin secretion [20]. Presumably has GLDH an anabolic function in leukocytes contrary to other organs, particularly the liver [21]. The GLUD1 gene (for housekeeping GLDH) is localized on human chromosome 10 and is present as thermo stable; GLUD2, however, (for nerve tissue-specific) is localized on human chromosome $\mathrm{X}$ and is present as a thermo labile isoprotein [5] [6] [20]. GLUD 1 is mainly present in hepatocytes, whereas GLUD 2 is in nerve tissues, testicles, retina and leukocytes [2] [22] [23]. There are no other relevant data on GLDH activity in leukocytes except its role in defining of neurodegenerative disorders.

GLDH activity was partly deficient in the brain, particularly in the leukocytes of patients with heterogeneous neurological disorders and the degeneration of multiple neuronal systems. The determination of GLDH activity in leukocytes is 
the only and indirect way for its activity validation in the brain as is the GLDH from brain and leukocytes mainly presented through GLUD 2 [18] [24] [25]. The diminished GLDH activity is presumably systemic-metabolic damage, but expressed as a brain disease because of high glutamate concentrations in the brain [22] [27]. Partial GLDH deficiency is not a sufficient indicator of an obvious neurological disease; however, it may play an indirect role in the development and course of neurodegenerative diseases [22] [26]. Glutamate metabolism appeared preferentially regulated by a control of GLDH expression rather than glutamine synthetase of both astrocytic enzymes [27] [28]. Urea, threonine, glutamate, citrulline, alpha-aminobutyric acid, ornithine, ammonia and arginine are elevated in the cerebrospinal fluid of patients with mild cognitive impairment and Alzheimer's disease [29]. The increased GLDH activities was found in patients with tuberculosis and pyogenic meningitis, but not in patients with brain tumours according to control activities [30] using the unsuitably unspecific method for GLDH determination. It is well known that the GLDH activity is partly deficient in the brain, particularly in the leukocytes of patients with heterogeneous neurological disorders and degeneration of multiple neuronal systems. In olivopontocerebellar atrophy, atypical Parkinson's disease, Alzheimer disease and bulbar palsy the total and thermo stable GLDH are diminished but in motor neural degeneration is diminished total and in cerebellar ataxia only thermo stable GLDH [3] [6] [22] [26] [31]. Therefore is a determination of leukocytes GLDH activity recommended in differential diagnostics of neurodegenerative disorders.

Somehow lower GLDH activity in leukocytes of alcoholics and specially its fast increase after cessation of drinking are not a sufficient indicators of an obvious neurodegenerative disorder caused by regular and/or excessive alcohol consumption, yet, it may play an indirect role in the development and course of neurodegenerative alcohol induced diseases. Like the alcohol dementia as an outcome of alcohol abuse or the greater exposure to infection because of diminished antibody protein production [21].

\section{GLDH Activity in Leukocytesin Aging}

In the brain the normally excitotoxic mechanism eliminates worn-out neuron branches from dendrites to enable new sprouts. The pathological excitotoxic mechanism is thought to be caused by processes, such as inadequate uptake, pathological metabolism in glial cells or release from terminals, and triggers excessive glutamate activity [8] [31]. The accumulation of glutamate and degeneration of postsynaptic neurons was found post-mortem in patients with neurodegenerative diseases of cerebellum and basal ganglia in patients who had GLDH deficiency in leukocytes and brain [23]. Many healthy subjects have a very low leukocyte GLDH activity [19]. In men, GLDH activity declines almost evenly through the ages; however, in women it declines faster in the first five decades. In the years of menopause, GLDH activity declines slower. Overall GLDH activity decreases more slowly in the age-group from 30 to 60 years, yet evidently 
more rapidly afterwards. The metabolism of proteins and possibly also of carbohydrates in leukocytes probably diminish with ageing. The diminished GLDH activity in leukocytes and the brain vary considerably, but they are parallel with neurodegenerative diseases. The gradually decreased GLDH activity over life may have some contribution in the process of aging. GLDH activity in leukocytes diminishes with ageing in both genders, particularly after the age of 50. It is assumed that the same process takes place also in the brain. It was presumed that the gradual decrease in GLDH activity is a normal process of growing older. Nevertheless, in the cases of neurodegenerative disorders is the rapidity of gradual GLDH activity decline faster. The fastest decline is perhaps in GLDH activity after 60 years of age [32].

\section{GLDH Activity in the Cerebrospinal Fluid}

The damage to the cells of the central nervous system leads to enzyme release and to a rise of enzyme activities in the extracellular fluid. However, the blood-cerebrospinal fluid barrier is normally impermeable to enzymes. Enzyme elevation is for the most part only detectable in the cerebrospinal fluid [33]. Expectations regarding the provision of special enzymes in cerebrospinal fluid, derived from nerve cells or from structures of cerebrospinal fluid spaces, have not yet been fulfilled. Nevertheless, such a discovery would be very important for diagnostics [34].

The cerebrospinal fluid is the first barrier in the protection of the brain against ammonia [1]. However, to our knowledge there is no relevant data about the GLDH activity in cerebrospinal fluid. We wanted to determine whether there is any measurable GLDH activity and any differences in the cerebrospinal fluid of patients with and without neurological diseases. In experimental study about regulation of glutamate level in rat brain through activation of GLDH with protopine and extracts of medical plants the results suggests a possibility to regulate glutamate level in human brain through activation of GLDH, too. In traditional Chinese medicine was Corydalis cava which contains protopine used as medicine for treatment of neurodegenerative diseases [35] [36].

\section{Subjects and Methods}

The study was carried out on the inpatients at the Department of Neurology of the Maribor University Medical Centre. The patients were hospitalized for the regular diagnostic purposes in the department of neurology. They were grouped according to the usual classification of neurological diseases: Degenerative (Parkinson's, Alzheimer's, Huntington's diseases, Amyotrophic Lateral Sclerosis), Demyelinating (multiple sclerosis, Devic disease, Balo's disease), Inflammatory (neuritis, polyneuritis, polyradiculitis), Vascular diseases (stroke, haemorrhage) and Other neurological diseases (epilepsy, infectious diseases). All patients were subjected to complete clinical investigation including neurological examination, cerebrospinal fluid analysis, brain CT or MRI and blood examinations. Informed consent was obtained for both diagnostic and medical research purposes. 
Many patients complained of various neurological symptoms, mainly vertigo, paraesthesia and headache, but no neurological disease was confirmed. These were denoted as the patients without neurological diseases. Included were all the patients, directed for a hospitalization, with an evident neurological signs and excluded that ones without them.

GLDH was determined to be present in cerebrospinal fluid, blood serum and blood leukocytes. We also determined all routine markers. The blood and cerebrospinal fluid samples were taken once during a regular diagnostic procedure as the take ones cerebrospinal fluid is a very invasive intervention.

For defining GLDH activity in leukocytes, we modified several methods to develop our own. Leukocytes were isolated from venous blood with the Skoog and Beck method, followed by two freeze-thaw cycles and the addition of Triton $\mathrm{X}-100$ at $-20^{\circ} \mathrm{C}[20]$.

The catalytic GLDH activities were determined with the new standard method proposed by the German Society of Clinical Chemistry (GSCC) in 1992 [35].

The thermal inactivation of GLDH in serum was performed with serum heating at $47.5^{\circ} \mathrm{C}$ for one hour [37]. We calculated the percentage of GLDH inactivation, with the corresponding thermolabile isoenzyme. We took the $20 \%$ barrier of thermo stable as the lowest reliable percentage [4] of GLDH inactivation; this reflects the findings of Grossman, as he was able to inactivate approximately $17 \%$ of soluble (thermostabile) and approximately $49 \%$ of particulate (thermo labile) GLDH [38].

Statistical analysis was carried out with the use of SPSS 16.0.1 for Windows. Frequencies, arithmetic mean, median, variance, standard deviation and standard error of the arithmetic mean, highest and lowest values, specificity and sensitivity, Pearson Chi-Square test, nonparametric Mann-Whitney U, Wilcoxon W tests and Spearman's rho, Pearson Correlation tests were used.

\section{Results and Discussion}

GLDH activity has been researched in the cerebrospinal fluid, serum and leukocytes of 91 subjects: 41 (45.05\%) women and 50 (54.95\%) men (Table 1). GLDH activity has been researched in the cerebrospinal fluid, serum and leukocytes of 97 subjects: 44 (45.36\%) women and 53 (54.64\%) men. The majority of patients with neurological diseases had demyelinating diseases (Table 2).

Table 1. General data.

\begin{tabular}{cccccccccc}
\hline & all & $\%$ & men & $\%$ & women & $\%$ & GLDH LQ & GLDH S & GLDH L \\
\hline $\begin{array}{c}\text { Without } \\
\text { neurl.dis. }\end{array}$ & 35 & 38.46 & 18 & 51.43 & 17 & 48.57 & 35 & 32 & 30 \\
$\begin{array}{c}\text { With } \\
\text { neurol.dis. }\end{array}$ & 56 & 62.54 & 32 & 57.14 & 24 & 42.86 & 56 & 56 & 46 \\
Total & 91 & 100.00 & 50 & 100.00 & 41 & 100.00 & 91 & 88 & 76 \\
\hline
\end{tabular}

Note: $\mathrm{LQ}=$ cerebrospinal fluid, $\mathrm{S}=$ serum, $\mathrm{L}=$ leukocytes, neurol. dis. = neurological disorder. 
Table 2. General data about diagnostic groups with neurological diseases.

\begin{tabular}{ccc}
\hline Diagnostic group & $\mathrm{n}$ & $\%$ \\
\hline All with neurological diseases & 62 & 100.00 \\
Demyelinating diseases & 20 & 32.26 \\
Degenerative disorders & 12 & 19.35 \\
Vascular diseases & 11 & 17.74 \\
Inflammatory diseases & 11 & 17.74 \\
Other neurological diseases & 8 & 12.90 \\
\hline
\end{tabular}

The reference values were determined with a deductive method in the group of 35 neurologically healthy patients. Using the non-parametric statistical analysis for determining reference ranges, the interval of 5- and 95-percentiles was chosen. The reference interval from 8.0 - $106 \mathrm{nkat} / \mathrm{L}$ was calculated.

We did not find statistically significant differences of GLDH activity in serum and leukocytes between the patients with and without diseases. But the very low leukocyte GLDH activity in patients with neurodegenerative diseases confirmed the Plaitakis findings (Figure 1, Tables 3-5). However, the lowest values were measured in patients with neurological diseases and unexpected in patients with degenerative and inflammatory disorders. We found a statistically significant difference of GLDH activity in the cerebrospinal fluid in these two groups of patients [3] [6] [22] [26] [31].

However, four patients, one with an inflammatory disorder and three with demyelinating diseases, have no measurable GLDH activity in the cerebrospinal fluid.

Two thirds of patients with degenerative disorders and more than one third of patients with inflammatory disorders had statistically significantly reduced GLDH activities. Among those with degenerative disorders, only one patient had a GLDH activity ( 16 nkat/L) on the bottom end of the normal level.

The GLDH activity of patients with degenerative disorders is exceedingly lower in comparison with other neurological diseases. Even the span between lowest and highest activity was much lower (Figure 1). The elimination of glutamate as an excitatory neurotransmitter consequently lowers the neuro-excitotoxic process, which presumably leads to neuro-degeneration. The neuro-excitotoxic process at degenerative disorders is probably even quicker.

However, most, if not all, dorsal root ganglion neurons use the neurotransmitter glutamate [17] [39]. An excess of glutamate is implicated in cases of peripheral neuropathies as well [40]. The increased pain sensation, following tissue and nerve injury in arthritis, myalgia, and tendonitis, elevates glutamate levels in affected tissues [17] [41]. We found statistically significant lower GLDH activity in cerebrospinal fluid in patients with inflammatory disorders (Mann-Whitney $\mathrm{U}=105.500, \mathrm{Z}=-2.242, p=0.02$ ). This indicates that decreased GLDH activity could possibly cause an excess of glutamate in peripheral neuropathies in a similar way as in neurodegenerative diseases. This is an absolutely new discovery that needs further investigation. 


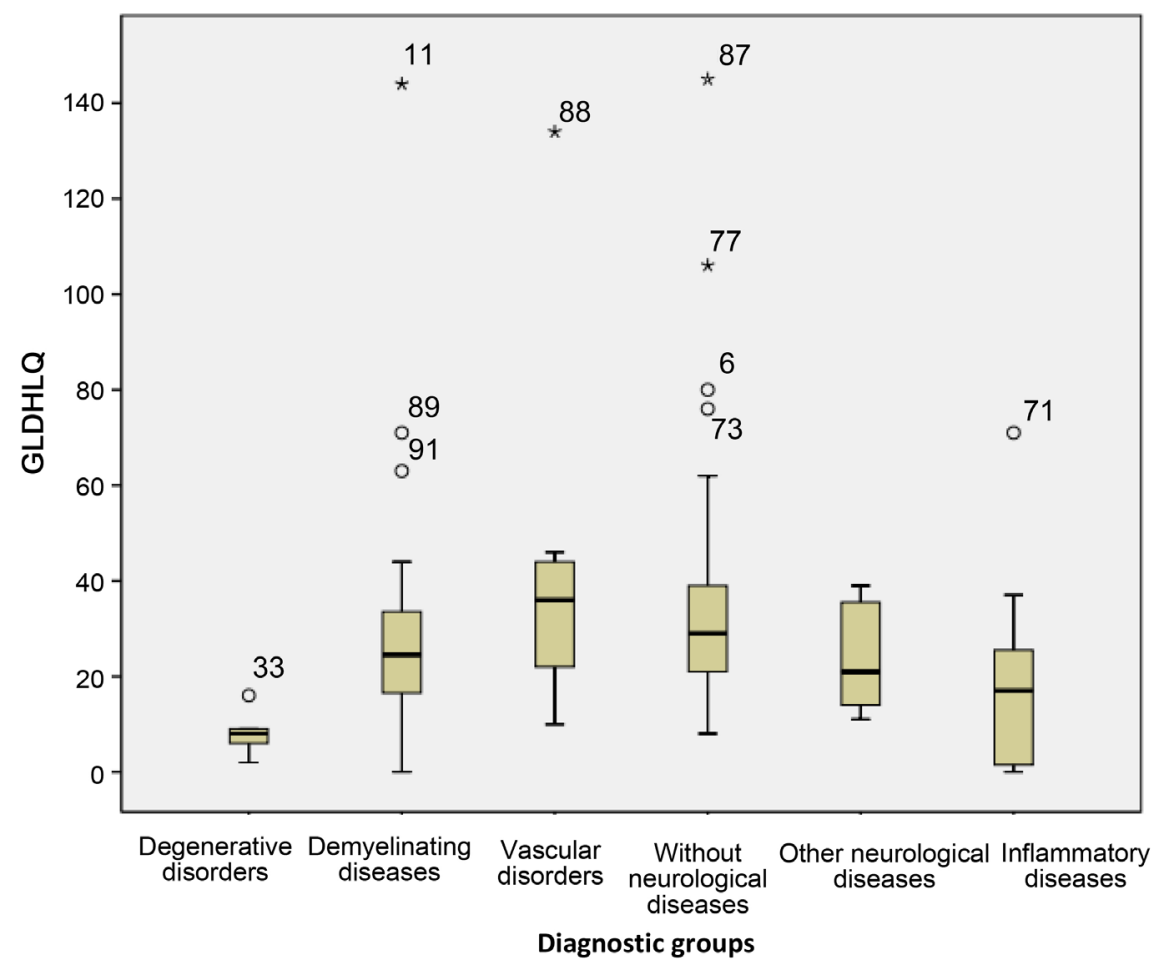

Figure 1. Differences of GLDH values (nkat/L) in cerebrospinal fluid according to diagnostic groups. There was a statistically significant difference for the Degenerative disorders and Inflammatory Diseases groups. Note: The distribution is displayed with a box plot graph (central line indicates median, boxes $1^{\text {st }}$ and $3^{\text {rd }}$ third part, handles $1^{\text {st }}$ and $9^{\text {th }}$ tenth part, o deviation $=1.5-3 \mathrm{x}$ height of a box, ${ }^{*}$ extreme value $\geq 3 \mathrm{x}$ height of a box.

Table 3. Statistical data of age, GLDH values in cerebrospinal fluid (nkat/L), sera (nkat/L) and leukocytes $(\mu \mathrm{kat} / \mathrm{g})$ of neurological healthy patients and patients with neurological diseases.

\begin{tabular}{ccccccccc}
\hline & $\begin{array}{c}\text { Age } \\
(\mathrm{H})\end{array}$ & $\begin{array}{c}\text { GLDH LQ } \\
(\mathrm{H})\end{array}$ & $\begin{array}{c}\text { GLDH } \\
\text { S (H) }\end{array}$ & $\begin{array}{c}\text { GLDH } \\
\text { L }(\mathrm{H})\end{array}$ & $\begin{array}{c}\text { Age } \\
(\mathrm{D})\end{array}$ & $\begin{array}{c}\text { GLDH LQ } \\
(\mathrm{D})\end{array}$ & $\begin{array}{c}\text { GLDH } \\
\text { S (D) }\end{array}$ & $\begin{array}{c}\text { GLDH L } \\
(\mathrm{D})\end{array}$ \\
\hline Mean & 47.18 & 36.54 & 168.75 & 1.528 & 51.22 & 26.73 & 109.11 & 1.468 \\
Median & 42.50 & 29.00 & 63.00 & 1.410 & 52.00 & 21.00 & 78.00 & 1.380 \\
Variance & 221.04 & 832.138 & $168,243.31$ & 0.601 & 251.768 & 809.791 & $18,868.419$ & 0.200 \\
Std. Dev. & 14.87 & 28.85 & 410.18 & 0.78 & 15.87 & 28.46 & 137.36 & 0.45 \\
Minimum & 20 & 8 & 13 & 0.23 & 18 & 0 & 11 & 0.46 \\
Maximum & 90 & 145 & 2213 & 3.96 & 80 & 144 & 839 & 2.51 \\
\hline
\end{tabular}

Note: $\mathrm{LQ}=$ cerebrospinal fluid, $\mathrm{S}=$ serum, $\mathrm{L}=$ leukocytes, $(\mathrm{H})=$ patients without neurological diseases, $(\mathrm{D})$ $=$ patients with neurological diseases, Std. Dev. = standard deviation.

Table 4. Statistical data on cerebrospinal fluid, leukocyte and serum GLDH activity.

\begin{tabular}{cccc}
\hline & GLDH LQ & GLDH S & GLDH L \\
\hline Mann-Whitney U & 728.000 & 843.500 & 603.500 \\
Wilcoxon W & 2324.000 & 1371.500 & 1068.500 \\
Z & -2.057 & -0.455 & -0.919 \\
p (2-tailed) & $0.040^{*}$ & 0.649 & 0.358
\end{tabular}

Note: $\mathrm{LQ}=$ cerebrospinal fluid, $\mathrm{S}=$ serum, $\mathrm{L}=$ leukocytes, ${ }^{*}=$ statistically significant. 
Table 5. Statistical data of GLDH (nkat/L) values in cerebrospinal fluid according to diagnostic groups.

\begin{tabular}{|c|c|c|c|c|c|c|}
\hline GLDH LQ & $\begin{array}{l}\text { Degenerative } \\
\text { disorders }\end{array}$ & $\begin{array}{c}\text { Demye-linating } \\
\text { diseases }\end{array}$ & $\begin{array}{l}\text { Vascular } \\
\text { disorders }\end{array}$ & $\begin{array}{c}\text { Inflammatory } \\
\text { disorders }\end{array}$ & $\begin{array}{c}\text { Other } \\
\text { neurological } \\
\text { diseases }\end{array}$ & $\begin{array}{c}\text { Without } \\
\text { neurological } \\
\text { diseases }\end{array}$ \\
\hline Mean & 8.17 & 31.65 & 40.09 & 19.64 & 23.88 & 36.54 \\
\hline Median & 8.00 & 24.50 & 36.00 & 17.00 & 21.00 & 29.00 \\
\hline Variance & 20.967 & 1024.555 & 1142.291 & 449.655 & 128.696 & 832.138 \\
\hline Std. Dev. & 4.58 & 32.01 & 33.80 & 21.21 & 11.34 & 28.85 \\
\hline Minimum & 2 & 0 & 10 & 0 & 11 & 8 \\
\hline Maximum & 16 & 144 & 134 & 71 & 39 & 145 \\
\hline Mann-Whitney U & 10.000 & 292.000 & 169.500 & 105.500 & 104.000 & \\
\hline Z & -3.508 & -1.015 & -0.593 & -2.242 & -1.124 & \\
\hline $\mathrm{P}$ & $0.000^{*}$ & 0.310 & 0.553 & $0.025^{\star}$ & 0.261 & \\
\hline
\end{tabular}

Note: $\mathrm{LQ}=$ cerebrospinal fluid, Std. Dev. $=$ standard deviation, ${ }^{*}=$ statistically significant.

We have confirmed the presence of both thermostable and thermolabile isoenzymes in human cerebrospinal fluid, but we did not find substantial differences in temperature inactivation between the groups. A statistically significant correlation was also found between total and inactivated GLDH in the cerebrospinal fluid and serum in patients with neurological diseases. The GLDH activity in cerebrospinal fluid is probably more decreased, because it is mitochondrial rather than GLDH from the rough endoplasmic reticulum [4] [5] [42] [43].

These studies indicate that the decrease in GLDH activity in the cerebrospinal fluid of patients with neurodegenerative disorders may be one of the reasons for the neuro-excitotoxic glutamate effect. The role of decreased GLDH activity in patients with inflammatory disorders needs further investigation to be adequately understood.

\section{Conclusions}

Obviously GLDH has a significant role in regulation of the cell metabolism and in the elimination of possible excitotoxic glutamate neurotransmitter from synaptic cleft. The evaluation is possible only with indirect measurements through its activity in leukocytes and in the cerebrospinal fluid. In normal life, the gradual decrease in GLDH activity may be one of key factors for neurodegenerative ageing processes. Thus it may play an indirect role in the development and course of aging of the brain. As partial GLDH deficiency is not a sufficient indicator of an obvious neurological disease, however, it may play an indirect role in the development and course of neurodegenerative diseases such as olivopontocerebellar atrophy, atypical Parkinson's disease, Alzheimer disease and bulbar palsy. Defining its activity in leukocytes is at the moment the only valuable experimental method. The second one could be the measurement in cerebrospinal fluid with its limitations because of invasive proceeding. The results suggest a possibility to regulate glutamate level in human brain through activation of GLDH. 
The activity of GLDH in leukocytes and in the cerebrospinal fluid is our further research focus, particularly in the field of differential diagnostics of neurodegenerative diseases.

\section{Conflict of Interest}

Both authors have none declared conflict of interest.

\section{Ethical Standards Statement}

All procedures followed were in accordance with the ethical standards of the responsible committee on human experimentation (institutional and national) and with the Helsinki Declaration of 1975, as revised in 2008.

\section{Informed Consent}

Informed consent was obtained from all patients for being included in the study.

\section{References}

[1] Thomas, L. (2005) Glutamate-Dehydrogenase. In: Thomas, L., Ed., Labor und Diagnose, 6th Edition, TH-Books, Frankfurt/Main, 103-106.

[2] Hussain, M.M., Zannis, V.I. and Plaitakis, A. (1989) Characterization of Glutamate Dehydrogenase Isoproteins Purified from the Cerebellum of Normal Subjects and Patients with Degenerative Neurological disorders, and from Human Neoplastic Cell Lines. The Journal of Biological Chemistry, 264, 20730-20735.

[3] Lee, W.K., Shin, S., Cho, S.S. and Park, J.S. (2000) Purification and Characterization of Glutamate Dehydrogenase as Another Isoprotein Binding to the Membrane of Rough Endoplasmic Reticulum. Journal of Cellular Biochemistry, 76, 244-253. https://doi.org/10.1002/(SICI)1097-4644(20000201)76:2<244::AID-JCB8>3.0.CO;2-K

[4] Kravos, M. and Malešič, I. (2008) Kinetics and Isoforms of Serum Glutamate Dehydrogenase in Alcoholics. Alcohol, 43, 281-286.

https://doi.org/10.1093/alcalc/agn010

[5] Plaitakis, A. and Zaganas, I. (2001) Regulation of Human Glutamate Dehydrogenases: Implications for Glutamate, Ammonia and Energy Metabolism in Brain. Journal of Neuroscience Research, 66, 899-908. https://doi.org/10.1002/jnr.10054

[6] Shashidharan, P., Clarke, D.D., Ahmed, N., Moschonas, N. and Plaitakis A. (1997) Nerve Tissue-Specific Human Glutamate Dehydrogenase That Is Thermolabile and Highly Regulated by ADP. Journal of Neurochemistry, 68, 1804-1811. https://doi.org/10.1046/j.1471-4159.1997.68051804.x

[7] Hassel, B. and Dingledine, R. (2006) Glutamate. In: Siegel, G.J., Albers, R.W., Brady, S.T. and Price, D.L., Eds., Basic Neurochemistry, 7th Edition, Elsevier Academic press, Amsterdam, 267-271.

[8] Stahl, S. (2000) Psychosis and Schizophrenia, In: Stahl, S., Ed., Essential Psychopharmacology, Cambridge University Press, Cambridge, 365-399.

[9] Hörtnagl, H. and Hellweg, R. (1997) Pathophysiological Aspects of Human Neurodegenerative Diseases. Wiener klinische Wochenschrift, 109, 623-635.

[10] Yoon, H.Y., Hwang, S.H., Kim, U., Hwang, O., Kim, D., Choi, Y. and Cho, S.W. (2002) Decreased Expression of Glutamate Dehydrogenase by Prolonged Intake of Monosodium Glutamate in Rat Brain. Experimental Brain Research, 142, 297-300. https://doi.org/10.1007/s00221-001-0961-0 
[11] Michaelis, E.K., Wang, X., Pal, R., Bao, X., Hascup, K.N., Wang, Y., Wang, W.T., Hui, D., Agbas, A., Choi, I.Y., Belousov, A. and Gerhardt, G.A. (2011) Neuronal Glud1 (Glutamate Dehydrogenase 1) Over-Expressing Mice: Increased Glutamate Formation and Synaptic Release, Loss of Synaptic Activity, and Adaptive Changes in Genomic Expression. Neurochemistry International, 4, 473-481. https://doi.org/10.1016/j.neuint.2011.03.003

[12] Carlton, S.M. (2001) Peripheral Excitatory Amino Acids. Current Opinion in Pharmacology, 1, 52-56. https://doi.org/10.1016/S1471-4892(01)00002-9

[13] Garry, E.M., Jones, E. and Fleetwood-Walker, S.M. (2004) Nociception Invertebrates: Key Receptors Participating in Spinal Mechanisms Ofchronic Pain in Animals. Brain Research Reviews, 46, 216-224. https://doi.org/10.1016/j.brainresrev.2004.07.009

[14] Willis, W.D.J. and Coggeshall, R.E. (2004) Sensory Mechanisms of the Spinal Cord. 3rd Edition, Plenum, New York. https://doi.org/10.1007/978-1-4615-0037-7

[15] Watkins, J.C. (2000) L-Glutamate as a Central Neurotransmitter: Looking Back. Biochemical Society Transactions, 28, 297-309. https://doi.org/10.1042/bst0280297

[16] McKenna, M.C., Stridh, M.H., McNair, L.F., Sonnewald, U., Waagepetersen, H.S. and Schousboe, A. (2016) Glutamate Oxidation in Astrocytes: Roles of Glutamate Dehydrogenase and Aminotransferases. Journal of Neuroscience Research, 94, 1561-1571. https://doi.org/10.1002/jnr.23908

[17] Miller, K.E., Hoffman, E.M., Sutharshan, M. and Schechter, R. (2011) Glutamate Pharmacology and Metabolism in Peripheral Primary Afferents: Physiological and Pathophysiological Mechanisms. Pharmacology \& Therapeutics, 130, 283-309. https://doi.org/10.1016/j.pharmthera.2011.01.005

[18] Hugon, J., Tabaraud, F., Rigaud, M., Vallat, J.M. and Dumas, M. (1989) Glutamate Dehydrogenase and Aspartate Aminotransferase in Leukocytes of Patients with Motor Neuron Disease. Neurology, 39, 956-958. https://doi.org/10.1212/WNL.39.7.956

[19] Kravos, M., Kralj, G. and Malešič, I. (2008) Encimi v likvorju. Slovenian Medical Society, 77, 593-599.

[20] Devlin, T.M. (2001) Textbook of Biochemistry with Clinical Correlations. Wiley \& Sons, New York, 784-785, 895-896.

[21] Kravos, M. and Malešič, I. (2010) Changes in Leukocyte Glutamate Dehydrogenase Activity in Alcoholics upon Break in Alcohol Consumption. Clinical Biochemistry, 43, 272-277. https://doi.org/10.1016/j.clinbiochem.2009.10.011

[22] Yamaguchi, T., Hayashi, K., Murakami, H., Ota, K. and Maruyama, S. (1982) Glutamate Dehydrogenase Deficiency in Spinocerebellar Degenerations. Neurochemical Research, 7, 627-636. https://doi.org/10.1007/BF00965128

[23] Plaitakis, A. and Berl, S. (1983) Oral Glutamate Loading in Disorders with Spinocerebellar and Extrapyramidal Involvement: Effect on Plasma Glutamate, Aspartate and Taurine. Journal of Neural Transmission, 19, 65-74.

[24] Plaitakis, A., Latsoudis, H., Kanavouras, K., Ritz, B., Bronstein, J.M., Skoula, I., Mastorodemos, V., Papapetropoulos, S., Borompokas, N., Zaganas, I., Xiromerisiou, G., Hadjigeorgiou, G.M. and Spanaki, C. (2010) Gain-of-Function Variant in GLUD2 Glutamate Dehydrogenase Modifies Parkinson's Disease Onset. European Journal of Human Genetics, 18, 336-341. https://doi.org/10.1038/ejhg.2009.179

[25] Trofimova, L.K., Araújo, W.L., Strokina, A.A., Fernie, A.R., Bettendorff, L. and Bunik, V.I. (2012) Consequences of the $\alpha$-Ketoglutarate Dehydrogenase Inhibition for Neuronal Metabolism and Survival: Implications for Neurodegenerative Diseases. Current Medicinal Chemistry, 19, 5895-5906. 
https://doi.org/10.2174/092986712804143367

[26] Iwatsuji, K., Nakamura, S. and Kameyama, M. (1989) Lymphocyte Glutamate Dehydrogenase Activity in Normal Aging and Neurological Diseases. Gerontology, 35, 218-224. https://doi.org/10.1159/000213026

[27] Hardin, H., Bernard, A., Rajas, F., Fevre-Montange, M., Derrington, E., Belin, M.F. and Didier-Bazes, M. (1994) Modifications of Glial Metabolism of Glutamate after Serotonergic Neuron Degeneration in the Hippocampus of the Rat. Molecular Brain Research, 26, 1-8. https://doi.org/10.1016/0169-328X(94)90067-1

[28] Hardin-Pouzet, H., Krakowski, M., Bourbonnière, L., Didier-Bazes, M., Tran, E. and Owens, T. (1997) Glutamate Metabolism Is Down-Regulated in Astrocytes during Experimental Allergic Encephalomyelitis. Glia, 20, 79-85. https://doi.org/10.1002/(SICI)1098-1136(199705)20:1<79::AID-GLIA8>3.0.CO;2-0

[29] Kaiser, E., Schoenknecht, P., Kassner, S., Hildebrandt, W., Kinscherf, R. and Schroeder, J. (2010) Cerebrospinal Fluid Concentrations of Functionally Important Amino Acids and Metabolic Compounds in Patients with Mild Cognitive Impairment and Alzheimer's Disease. Neurodegenerative Diseases, 7, 251-259. https://doi.org/10.1159/000287953

[30] Far, S.R. and Millimoria, F.R. (1996) Levels of Glutamic Acid Decarboxylase (GAD), Gamma Amino Butyric Acid Transaminase (GABA-T), Glutamic Acid Dehydrogenase (GLDH) and Proteins in Cerebrospinal Fluid of Certain Neurological Disorders. Indian Journal of Medical Sciences, 50, 99-102.

[31] Plaitakis, A. (1990) Glutamate Dysfunction and Selective Motor Neuron Degeneration Amyotrophic Lateral Sclerosis: A Hypothesis. Annals of Neurology, 28, 3-8. https://doi.org/10.1002/ana.410280103

[32] Kravos, M. and Malešič, I. (2010) Glutamate Dehydrogenase Activity in Leukocytes and Ageing. Neurodegenerative Diseases, 7, 239-242. https://doi.org/10.1159/000273582

[33] Schmidt, E. and Schmidt, F. (1967) Guide to Practical Enzyme Diagnosis. Boeringer Mannheim GmbH, Mannheim, 105.

[34] Dommasch, D. and Mertens, H.G. (1980) Cerebrospinalfluessigkeit-CSF. Georg Thieme Verlag, Stuttgart, New York, 81.

[35] Lee, K.H., Huh, J.W., Choi, M.M., Yoon, S.Y., Yang, S.J., Hong, H.N. and Cho, S.W. (2005) Regulation of Glutamate Level in Rat Brain through Activation of Glutamate Dehydrogenase by Corydalis ternata. Experimental \& Molecular Medicine; 37, 371-377. https://doi.org/10.1038/emm.2005.47

[36] Adsersen, A., Kjølbye, A., Dall, O. and Jäger, A.K. (2007) Acetylcholinesterase and Butyrylcholinesterase Inhibitory Compounds from Corydalis cava Schweigg. \& Kort. Journal of Ethnopharmacology, 113, 179-182. https://doi.org/10.1016/j.jep.2007.05.006

[37] Working Group on Enzymes (1992) Proposal of Standard Methods for the Determination of Enzyme Catalytic Concentrations in Serum and Plasma at 37 Degrees C. III. Glutamate Dehydrogenase ( $L$-Glutamate: $\mathrm{NAD}(\mathrm{P})^{+}$Oxidoreductase (Deaminating), EC 1.4.1.3). European Journal of Clinical Chemistry and Clinical Biochemistry, 30, 493-502.

[38] Grossman, A., Rosenberg, R.N. and Warmoth, L. (1987) Glutamate and Malate Dehydrogenase Activities in Joseph Disease and Olivopontocerebellar Atrophy. Neurology, 37, 106-111. https://doi.org/10.1212/WNL.37.1.106

[39] Hoffman, E.M., Schechter, R. and Miller, K.E. (2010) Fixative Composition Alters Distributions of Immunoreactivity for Glutaminase and Two Markers of Nocicep- 
tive Neurons, $\mathrm{Na}_{\mathrm{v}} 1.8$ and TRPV1, in the Rat Dorsal Root Ganglion. Journal of Histochemistry \& Cytochemistry, 58, 329-344.

https://doi.org/10.1369/jhc.2009.954008

[40] Carozzi, V.A., Canta, A., Oggioni, N., Ceresa, C., Marmiroli, P., Konvalinka, J., Zoia, C., Bossi, M., Ferrarese, C., Tredici, G. and Cavaletti, G. (2008) Expression and Distribution of "High Affinity" Glutamate Transporters GLT1, GLAST, EAAC1 and of GCPII in the Rat Peripheral Nervous System. Journal of Anatomy, 213, 539-546. https://doi.org/10.1111/j.1469-7580.2008.00984.x

[41] Xu, Q. and Yaksh, T.L. (2011) A Brief Comparison of the Pathophysiology of Inflammatory versus Neuropathic Pain. Current Opinion in Anaesthesiology, 24, 400-407. https://doi.org/10.1097/ACO.0b013e32834871df

[42] Plaitakis, A., Latsoudis, H. and Spanaki, C. (2011) The Human GLUD2 Glutamate Dehydrogenase and Its Regulation in Health and Disease. Neurochemistry International, 59, 495-509. https://doi.org/10.1016/j.neuint.2011.03.015

[43] Plaitakis, A., Zaganas, I. and Spanaki, C. (2013) Deregulation of Glutamate Dehydrogenase in Human Neurologic Disorders. Journal of Neuroscience Research, 91, 1007-1017. https://doi.org/10.1002/jnr.23176

\section{Scientific Research Publishing}

\section{Submit or recommend next manuscript to SCIRP and we will provide best} service for you:

Accepting pre-submission inquiries through Email, Facebook, LinkedIn, Twitter, etc. A wide selection of journals (inclusive of 9 subjects, more than 200 journals) Providing 24-hour high-quality service User-friendly online submission system Fair and swift peer-review system Efficient typesetting and proofreading procedure Display of the result of downloads and visits, as well as the number of cited articles Maximum dissemination of your research work

Submit your manuscript at: http://papersubmission.scirp.org/ Or contactwjins@scirp.org 